\title{
Gráfico de controle MCMAX para o monitoramento simultâneo do vetor de médias e da matriz de covariâncias
}

\author{
MCMAX control chart for the joint monitoring \\ of the mean vector and the covariance matrix
}

\author{
Antônio Fernando Branco Costa ${ }^{1}$ \\ Marcela Aparecida Guerreiro Machado ${ }^{1}$ \\ Fernando Antônio Elias Claro' ${ }^{1}$
}

\begin{abstract}
Resumo: $\mathrm{O}$ gráfico $T^{2}$ de Hotelling e o gráfico $|\mathrm{S}|$ da variância generalizada são utilizados para monitorar o vetor de médias e a matriz de covariâncias de processos multivariados. Neste artigo, propõe-se o uso de um único gráfico de controle para o monitoramento de processos bivariados, isto é, o gráfico de controle MCMAX cujo valor da estatística de monitoramento corresponde ao maior valor em módulo de quatro medidas amostrais das duas características de qualidade sob monitoramento, isto é, as suas médias e variâncias padronizadas. O usuário de gráficos de controle já está bem familiarizado com médias e variâncias amostrais; o mesmo não pode ser dito a respeito da estatística de Hotelling ou da variância generalizada. Conseqüentemente, ele preferirá usar o gráfico de controle proposto ao invés dos gráficos conjuntos de $T^{2}$ e $|\mathrm{S}|$. Além disso, o usuário, em geral, se sente mais seguro em intervir no processo somente após a ocorrência de um segundo ponto na região de ação do gráfico. Se o sinal for dado por dois pontos, não necessariamente vizinhos, porém próximos e na região de ação, o gráfico proposto terá um desempenho geral superior ao dos gráficos conjuntos de $T^{2} \mathrm{e}|\mathrm{S}|$ na detecção de desajustes do processo, exceto quando a correlação entre as duas características de qualidade for muito alta. Quando a correlação é muito alta e a causa especial desloca a média e/ou aumenta a variância de apenas uma das variáveis, $\mathrm{X}$ ou $\mathrm{Y}$, os gráficos de $T^{2}$ e $|\mathrm{S}|$ são, em geral, mais ágeis do que gráfico MCMAX.

Palavras-chave: Gráfico de controle MCMAX. Matriz de covariâncias. Vetor de médias. Processos bivariados. Regra especial de decisão.
\end{abstract}

\begin{abstract}
The control chart and the generalized variance $|S|$ chart are used for monitoring the mean vector and the covariance matrix of multivariate processes. In this article, we propose the use of a single chart for monitoring bivariate processes, that is, the MCMAX control chart based on a new statistic, which corresponds to the maximum among four sample values: the standardized sample means (in module) and the standardized sample variances (weighted). The sample means and sample variances are well known by the users; the same cannot be said for the Hotelling's statistic or the generalized variance. Consequently, they will prefer to use the proposed chart instead of the joint and $|S|$ charts. In general, the user prefers to wait until the occurrence of a second point beyond the control limit (not far from the first one) before interfering in the process. With this new rule, the proposed chart is faster in signaling out-of-control conditions, except when the correlation between the two quality characteristics is too high. In this case, the joint and $|S|$ charts are faster is signaling assignable causes that only affect one of the two quality characteristics, changing its mean and/or increasing its variance.
\end{abstract}

Keywords: MCMAX control chart. Covariance matrix. Mean vector. Bivariate processes. Special run rule.

\section{Introdução}

Os gráficos de Shewhart têm sido os dispositivos estatísticos mais utilizados no controle da qualidade de processos que requer o monitoramento de apenas uma característica de qualidade. Contudo, um número crescente de processos vem demandando o monitoramento simultâneo de várias características de qualidade. Neste contexto, os gráficos de Shewhart de $\overline{\mathrm{X}}$ e de $S^{2}$ são

substituídos pelo gráfico de $T^{2}$, proposto por Hotelling (1947), que serve para detectar alterações no vetor de médias de processos multivariados e pelo gráfico da variância generalizada $|S|$, proposto por Alt (1985), que serve para diagnosticar alterações na matriz de covariâncias.

\footnotetext{
${ }^{1}$ Departamento de Produção - FEG, Universidade Estadual Paulista "Júlio de Mesquita Filho"- UNESP, Av. Ariberto Pereira da Cunha, 333, CEP 12516-410, Guaratinguetá, SP, Brasil, E-mails: fbranco@ feg.unesp.br; marcela@ feg.unesp.br; fernandoclaro@uol.com.br
} 
Gráficos de controle mais eficientes que o gráfico $|\mathrm{S}|$ têm sido objeto de pesquisa. Costa e Machado (2008) propuseram para o monitoramento da matriz de covariâncias de processos bivariados um gráfico de controle que demanda apenas o cálculo das variâncias amostrais das duas características de qualidade. Os pontos no gráfico correspondem aos valores da estatística de VMAX, isto é, ao valor da maior variância amostral. O gráfico proposto por Costa e Machado (2008), denominado gráfico de VMAX, tem um desempenho superior ao do gráfico da variância generalizada $|S|$ e, além disso, tem uma melhor capacidade de diagnóstico, ou seja, com ele é mais fácil identificar a variável que teve sua variabilidade alterada pela ocorrência da causa especial. Machado, Magalhães e Costa (2008) estudaram as propriedades do gráfico de VMAX com amostragem dupla e Machado e Costa (2008) consideraram os gráficos de VMAX baseados no esquema EWMA (média móvel ponderada exponencialmente). Artigos que tratam do monitoramento simultâneo do vetor de médias e da matriz de covariâncias são poucos e recentes. Khoo (2005) propôs um gráfico de controle baseado nas estatísticas de $T^{2}$ e de $|\mathrm{S}|$ para o monitoramento de processos bivariados. A habilidade do gráfico proposto em sinalizar alterações no vetor de médias e/ou na matriz de covariâncias foi avaliada por meio de simulações. Os resultados obtidos não são animadores, pois o dispositivo é por demais lento na sinalização de desajustes do processo. Zhang e Chang (2008) propuseram dois gráficos de EWMA baseados em observações individuais que são capazes não só de detectar alterações no processo, mas também diagnosticar, com um bom índice de acerto, o tipo de alteração, se apenas no vetor de médias ou apenas na matriz de covariâncias, ou em ambos. A performance destes gráficos não foi comparada com a performance dos gráficos conjuntos de $T^{2}$ e $|\mathbf{S}|$. Chen, Cheng e Xie (2005) propuseram um gráfico de EWMA que é mais eficiente que os gráficos conjuntos de $T^{2}$ e $|\mathbf{S}|$ na sinalização de pequenas mudanças no processo.

$\mathrm{Wu}$ e Spedding (2000) foram os primeiros a considerarem a regra de dois pontos na região de ação, especificando ainda que, para se ter um alarme, o número de amostras após a ocorrência de um ponto na região de ação e antes da ocorrência do próximo ponto em tal região não deve exceder a $L$, sendo $L$ uma variável inteira cujo valor é estabelecido durante o projeto do gráfico de controle. Por exemplo, se o primeiro e o segundo ponto na região de ação são das amostras de número 100 e 104, respectivamente, o gráfico de controle sinalizará se $L \leq 3$, mas não se $L>3$. O grande interesse pelo uso da regra dos dois pontos pode ser explicado pelo fato de que muitos usuários preferem esperar a ocorrência de um segundo ponto na região de ação do gráfico antes de intervirem no processo (WU; SPEDDING, 2000a, b; WU; YEO; SPEDDING, 2001a, b; CALZADA; SCARIANO, 2001; DAVIS; WOODALL, 2002; COSTA; RAHIM,
2006; COSTA; MACHADO, 2007; COSTA; DE MAGALHÃES; EPPRECHT, 2009).

Neste artigo é proposto, para o monitoramento do vetor de médias e da matriz de covariâncias de processos bivariados, o uso de um único gráfico de controle baseado na estatística de MCMAX, que corresponde ao maior valor em módulo de quatro medidas das duas características de qualidade sob monitoramento, isto é, as suas médias e variâncias amostrais padronizadas. O gráfico proposto é denominado gráfico de MCMAX. Suas propriedades são obtidas considerando-se a regra de decisão de dois pontos na região de ação do gráfico.

A principal razão em se propor o gráfico de MCMAX é que ele é muito mais simples de ser utilizado se comparado com os gráficos de $T^{2}$ e $|\mathbf{S}|$, pois requer apenas o cálculo de estatísticas bem conhecidas pelo usuário, isto é, médias e variâncias amostrais.

\section{Descrição do gráfico maior valor em módulo}

Ao longo deste trabalho, assume-se que o gráfico MCMAX é utilizado para o controle de processos bivariados em que as características de qualidade, $X$ e $Y$, seguem uma distribuição normal com vetor de médias $\mu$ e matriz de covariâncias $\Sigma$ iguais a $\mu_{0}^{\prime}=\left(\mu_{\mathrm{x}} ; \mu_{\mathrm{y}}\right)$ e $\Sigma_{0}=\left(\begin{array}{cc}\sigma_{x}^{2} & \sigma_{x y} \\ \sigma_{x y} & \sigma_{y}^{2}\end{array}\right)$, respectivamente. A causa especial desloca o vetor de médias de $\mu_{0}$ para $\mu_{1}^{\prime}=\left(\mu_{x}+c \sigma_{x} ; \mu_{y}+d \sigma_{y}\right)$ e/ou altera a matriz de covariâncias de $\Sigma_{0}$ para: $\Sigma_{1}=\left(\begin{array}{cc}a^{2} \sigma_{x}^{2} & a b \sigma_{x y} \\ a b \sigma_{x y} & b^{2} \sigma_{y}^{2}\end{array}\right)$ ou para $\Sigma_{2}=\left(\begin{array}{cc}a^{2} \sigma_{x}^{2} & \sigma_{x y} \\ \sigma_{x y} & b^{2} \sigma_{y}^{2}\end{array}\right)$.

No primeiro caso, em que $\Sigma_{0}$ se altera para $\Sigma_{1}$, a causa especial não afeta a correlação entre $X$ e $Y$, que é dada por $\rho_{0}=\frac{\sigma_{x y}}{\sigma_{x} \sigma_{y}}$, no segundo caso, no entanto, a causa especial reduz a correlação de $\rho_{0}$ para: $\frac{\rho_{0}}{a b}$. Quando o gráfico MCMAX está em uso, amostras de tamanho $n$ são retiradas do processo em intervalos regulares de tempo e as características de qualidade correlacionadas $(X ; Y)$ são medidas. Com os dados da amostra, são calculadas as médias amostrais padronizadas, $Z_{x}=\sqrt{n}\left(\bar{X}-\mu_{x}\right) / \sigma_{x}$, $Z_{y}=\sqrt{n}\left(\bar{Y}-\mu_{y}\right) / \sigma_{y}$, e os desvios padrões amostrais ponderados e padronizados, $W_{x}=k S_{x} / \sigma_{x}$ e $W_{y}=k S_{y} / \sigma_{y}$. $\mathrm{O}$ ponto no gráfico MCMAX corresponde ao maior 
valor, em módulo, das quatro medidas amostrais, isto é, $\left(\left|Z_{x}\right|,\left|Z_{y}\right|, \mathrm{W}_{x}, \mathrm{~W}_{y}\right)$.

A constante de ponderação $k$ é determinada de tal sorte que, durante o período em controle, todas as quatro estatísticas $\left(\left|Z_{x}\right|,\left|Z_{y}\right|, \mathrm{W}_{x}, \mathrm{~W}_{y}\right)$ tenham a mesma probabilidade de assumirem valores maiores que $L C$, isto é, de excederem o limite de controle do gráfico MCMAX. O gráfico proposto sinaliza um desajuste no processo quando, ao menos um dos valores amostrais das quatro estatísticas $\left(\left|Z_{x}\right|,\left|Z_{y}\right|, \mathrm{W}_{x}, \mathrm{~W}_{y}\right)$, exceder o limite de controle, $L C$.

A implementação do gráfico de controle proposto é mais simples se comparado com a dos gráficos de $T^{2}$ e $|\mathbf{S}|$, uma vez que requer apenas o uso de estatísticas bem conhecidas pelo usuário, isto é, médias e variâncias amostrais.

O sistema de controle também é mais simples, pois com base em qual, ou quais, das estatísticas $\left(\left|Z_{x}\right|,\left|Z_{y}\right|\right.$, $\mathrm{W}_{r}, \mathrm{~W}_{y}$ ) excedem os limites de controle, é possível saber qual variável foi afetada pela causa especial. O mesmo não ocorre com os gráficos de $T^{2}$ e $|\mathbf{S}|$.

O desempenho do gráfico de MCMAX é viável quando se utiliza a regra especial de decisão de dois pontos na região de ação. Como o usuário normalmente não se sente seguro em intervir no processo após a ocorrência de um ponto na região de ação, o uso desta regra especial de decisão com o gráfico de MCMAX parece ser bem oportuno.

\section{Propriedades do gráfico maior valor em módulo}

$\mathrm{O}$ fato de as variáveis $Z_{\mathrm{x}}$ e $Z_{\mathrm{y}}$ serem independentes de $W_{\mathrm{x}}$ e de $W_{\mathrm{y}}$ facilita a obtenção das propriedades do gráfico MČMAX, isto é, o risco $\alpha$ de um alarme falso e o poder $P$ de detecção do gráfico. Com respeito às médias amostrais, a probabilidade $P_{\mathrm{M}}$ de $\left|Z_{x}\right|$ e/ou $\left|Z_{y}\right|$ exceder o limite de controle é dada pela Equação 1:

$$
P_{M}=1-\int_{L C-c \sqrt{n}}^{L C-c \sqrt{n}} \int_{L C-d \sqrt{n}}^{L C-d \sqrt{n}} f\left(Z_{x}, Z_{y}\right) d_{Z_{x}} d_{Z_{y}}(1)
$$

Com respeito aos desvios padrões, a probabilidade $P_{\mathrm{S}}$ de $W_{x}$ e/ou $W_{y}$ exceder o limite de controle é dada pela Equação $2^{y}$ (ver Apêndice):

$$
\begin{aligned}
& P_{S}=1-\int_{0}^{\frac{(n-1) L C}{k a^{2}}} \operatorname{Pr}\left[\chi_{n,\left(t \rho^{2} / 1-\rho^{2}\right)}^{2} \frac{(n-1) L C}{k b^{2}\left(1-\rho^{2}\right)}\right] \\
& \frac{1}{2^{(n-1) / 2} \Gamma[(n-1) / 2]} e^{-t / 2} t^{[(n-1) / 2]-1} d t
\end{aligned}
$$

em que $\chi_{n}^{2},\left(\rho^{2} / 1-\rho^{2}\right) \chi_{n}^{2}$ é a distribuição de qui-quadrado com $n$ graus de liberdade e parâmetro de não centralidade dado por $\left(\rho^{2} / 1-\rho^{2}\right) \chi_{n}^{2}$. Portanto a probabilidade $P$ do gráfico de controle dar um alarme é dada pela Equação 3:

$$
P=P_{\mathrm{M}}+P_{\mathrm{S}}-P_{\mathrm{M}} P_{\mathrm{S}}
$$

Estando em uso a regra de decisão de um ponto na região de ação, o limite de controle do gráfico proposto, $L C$, é obtido pela Equação 1, fazendo $c=d=0$ e $P_{M}=1-\sqrt{1-\alpha}$, sendo $\alpha$ definido a priori. A consequência de ordem prática associada ao risco $\alpha$ (alarme falso) é de uma intervenção no processo na hora errada, quando o mesmo está isento de causas especiais (o que em si já acarreta um custo - de interrupção do processo, de mão de obra - além de um risco de desajustar um processo que estava ajustado).

As sub-rotinas CSNDF e BNRDF, da biblioteca MICROSOFT (1995), foram utilizadas para o cálculo de probabilidades quando a distribuição é de qui-quadrado não central e quando ela é normal bivariada, respectivamente.

Quando a regra de decisão de um ponto na região de ação é substituída pela de dois pontos, torna-se mais difícil obter os limites do gráfico de controle, bem como o número médio de amostras necessárias para sinalizar um desajuste no processo, o NMA. Davis e Woodall (2002) modelaram uma cadeia de Markov com estados transientes e absorventes definidos pelas posições dos pontos amostrais no gráfico de controle. Conhecidas as propriedades desta cadeia de Markov, torna-se fácil obter os limites de controle e a expressão do NMA.

Estando em uso a regra de decisão de dois pontos na região de ação, o seguinte procedimento é então adotado na obtenção do $L C$ do gráfico MCMAX :

Com $L$ e $N M A_{0}$ dados, encontrar o valor de $Q_{0}$ tal que (Equação 4):

$$
s_{0}^{\prime} \times M \times \mathbf{1}=N M A_{0}
$$

em que $N M A_{0}$ é número médio de amostras entre alarmes falsos (especificado quando da construção do gráfico de controle), $\mathbf{M}$ é uma matriz de zeros exceto $m_{11}=Q_{0} ; m_{12}=-Q_{0} ; m_{\mathrm{i}, \mathrm{i}+1}=Q_{0}-1, i=1,2, \ldots, L ; \mathrm{e}$ $\mathrm{m}_{\mathrm{L}+1,1}=Q_{0}-1 ; \mathbf{l}$ é um $(L+1) \times 1$ vetor de uns; e $\mathrm{s}_{0}$ é um vetor $(L+1) \times 1 \mathrm{com} s_{1}=\left[1+(L-1) Q_{0}\right]^{-1}$ e s $=Q_{0} s_{1}, j=2, \ldots, L+1$. O limite de controle do grá́fico proposto, $L C$, é obtido da Equação 3 , fazendo $a=b=1, c=d=0$ e $P=1-Q_{0}$.

Quando o processo se desajusta ( $a$ ou $b$ assumem valores maiores do que um e/ou $c$ ou $d$ assumem valores diferentes de zero), o número médio de amostras necessárias para sinalizar tal desajuste é dado por

$$
N M A=s_{0}^{\prime} \times M \times \mathbf{1}
$$

em que $\mathbf{M}$ é agora uma matriz de zeros exceto para $m_{11}=Q ; m_{12}=-Q m_{\mathrm{i}, \mathrm{i}+1}=Q-1, i=1,2, \ldots, L ; \mathrm{e}$ $\mathrm{m}_{\mathrm{L}+1,1}=Q-1$, sendo $Q=1-P$ com $P$ dado pela Equação (3); e 1 é um vetor $(L+1) \times 1$ de uns. 
O programa para obtenção do limite de controle do gráfico MCMAX, bem como para o cálculo dos NMAs para diferentes mudanças nos parâmetros do processo, foi elaborado seguindo as instruções do parágrafo anterior. Para testá-lo, considerou-se primeiramente o gráfico de $X$ e, então, foram comparados os valores obtidos do NMA com aqueles encontrados na Tabela 2 do artigo de Davis e Woodall (2002). Depois de testado, o programa foi utilizado para estudar o efeito do parâmetro de projeto $L$ na performance do gráfico MCMAX, e para comparar o gráfico proposto com os gráficos de controle conjunto de $T^{2}$ e $|\mathrm{S}|$. A Tabela 1 mostra o efeito do parâmetro de projeto $L$ na performance do gráfico MCMAX. À medida que $L$ aumenta, a performance do gráfico melhora. A melhora é mais acentuada na faixa em que $L$ varia de 1 até 6. Por exemplo, se $L$ varia de 1 até 6 , considerando $a=b=1$ e $c=d=0,5$, o NMA diminui de 21,4 para 17,5 , o que representa uma redução percentual no valor do $N M A$ de aproximadamente $18,0 \%$. Por outro lado, se $L$ varia de 6 até 10, o NMA diminui de 5,2 para 5,1, o que representa uma redução percentual no valor do NMA de aproximadamente 2,0\%.

As Tabelas de 2 a 5 apresentam os NMAs do gráfico MCMAX e dos gráficos de controle conjunto de $T^{2}$ e $|\mathrm{S}|$ para diferentes perturbações nos parâmetros do processo. As Tabelas de 2 a 4 consideram que a causa especial altera a matriz de covariâncias de $\Sigma_{0}$ para $\Sigma_{1}$, enquanto que a Tabela 5 considera o caso em que $\Sigma_{0}$ muda para $\Sigma_{2}$. Na detecção de desajustes do processo, o gráfico proposto tem um desempenho geral superior ao dos gráficos conjuntos de $T^{2}$ e $|\mathrm{S}|$, exceto quando a correlação entre as duas características de qualidade é muito alta.

Quando a correlação é muito alta e a causa especial desloca a média e/ou a variabilidade de apenas uma das variáveis, $X$ ou $Y$, os gráficos de
$T^{2}$ e $|\mathbf{S}|$ são, em geral, mais ágeis do que o gráfico MCMAX. Quando a correlação é muito alta e, além disso, se altera com a presença da causa especial $\left(\Sigma_{0}\right.$ muda para $\Sigma_{2}$ ), os gráficos de $T^{2}$ e $|S|$ sinalizam esta condição de falta de controle com maior rapidez. A habilidade dos gráficos conjuntos de $T^{2}$ e $|\mathrm{S}| \mathrm{em}$ sinalizar um desajuste no processo é tanto melhor quanto maior for a correlação $\rho$ entre as variáveis $X$ e $Y$. A correlação $\rho$ tem pouca influência na performance do gráfico MCMAX.

\section{Exemplo}

Em uma linha de empacotamento de leite, a variável usual de monitoramento tem sido o volume de leite dentro dos saquinhos, medido indiretamente pelo peso. Um excesso de leite aumenta o risco do saquinho estourar durante o manuseio e transporte (COSTA; EPPRECHT; CARPINETTI, 2005). O monitoramento de uma variável adicional vem sendo proposto com o objetivo de melhor identificar os instantes em que o processo requer ajustes. Trata-se da espessura do saquinho de leite em sua parte superior, medida por meio de um dispositivo que the aplica uma leve pressão até que este fique estufado. As variáveis $X$ e $Y$, respectivamente, volume e espessura, são positivamente correlacionadas. $\mathrm{O}$ vetor de médias e a matriz de covariâncias são supostos conhecidos e iguais a $\mu_{0}{ }^{\prime}=(1000,0 \mathrm{~cm}$ cúbicos; 2,0 cm) e $\Sigma_{0}=\left(\begin{array}{cc}16,0 & 0,2 \\ 0,2 & 0,01\end{array}\right)$.

A Tabela 6 apresenta os valores de $X, Y, Z, W$ e MCMAX, para 10 amostras de tamanho 5, sendo que as 5 primeiras foram obtidas com o processo em controle, e as últimas cinco com a média e o desvio padrão de $X$ alterados, $\mu_{\mathrm{x}}=1004,0$ e $\sigma_{\mathrm{x}}=5,0$, isto é, $c=1$ e $a=1,25$. A correlação inicial de 0,5 passou

Tabela 1. Influência do parâmetro L no desempenho do gráfico $\operatorname{MCMAX}\left(\rho=0,5\right.$; matriz de covariâncias muda de $\Sigma_{0}$ para $\left.\Sigma_{1}\right)$.

\begin{tabular}{|c|c|c|c|c|c|c|c|c|c|c|c|c|c|c|}
\hline & & & & & \multicolumn{10}{|c|}{$n=5$} \\
\hline & & & & & \multicolumn{10}{|c|}{$L$} \\
\hline & & & & & 1 & 2 & 3 & 4 & 5 & 6 & 7 & 8 & 9 & 10 \\
\hline$a$ & $b$ & $c$ & $d$ & $L C=$ & 2,350 & 2,469 & 2,536 & 2,582 & 2,617 & 2,644 & 2,667 & 2,687 & 2,703 & 2,719 \\
\hline \multirow[t]{3}{*}{1,0} & 1,0 & 0 & 0 & - & 200,0 & 200,0 & 200,0 & 200,0 & 200,0 & 200,0 & 200,0 & 200,0 & 200,0 & 200,0 \\
\hline & & 0 & 0,5 & - & 45,3 & 40,9 & 39,1 & 37,9 & 37,3 & 36,8 & 36,3 & 36,1 & 35,9 & 35,7 \\
\hline & & 0,5 & 0,5 & - & 21,4 & 19,2 & 18,4 & 17,9 & 17,7 & 17,5 & 17,4 & 17,3 & 17,3 & 17,3 \\
\hline \multirow[t]{3}{*}{1,25} & 1,25 & 0 & 0 & - & 13,6 & 12,0 & 11,3 & 11,0 & 10,8 & 10,8 & 10,6 & 10,6 & 10,6 & 10,6 \\
\hline & & 0 & 0,5 & - & 8,9 & 7,8 & 7,3 & 7,1 & 7,0 & 7,0 & 6,9 & 6,9 & 6,9 & 6,9 \\
\hline & & 0,5 & 0,5 & - & 6,5 & 5,7 & 5,4 & 5,3 & 5,2 & 5,2 & 5,2 & 5,1 & 5,1 & 5,1 \\
\hline \multirow[t]{4}{*}{1,5} & 1,0 & 0 & 0 & - & 10,1 & 8,5 & 7,9 & 7,5 & 7,3 & 7,3 & 7,1 & 7,0 & 7,0 & 7,0 \\
\hline & & 0 & 0,5 & - & 7,6 & 6,5 & 6,1 & 5,9 & 5,8 & 5,8 & 5,7 & 5,7 & 5,7 & 5,7 \\
\hline & & 0,5 & 0 & - & 7,2 & 6,0 & 5,6 & 5,3 & 5,2 & 5,2 & 5,0 & 5,0 & 5,0 & 4,9 \\
\hline & & 0,5 & 0,5 & - & 5,8 & 5,0 & 4,7 & 4,5 & 4,4 & 4,4 & 4,3 & 4,3 & 4,3 & 4,3 \\
\hline
\end{tabular}


Tabela 2. Valores do NMA - gráficos conjuntos de $T^{2}$ e $|\mathrm{S}|$ e gráfico $\operatorname{MCMAX}\left(\rho=0,0\right.$; matriz de covariâncias muda de $\Sigma_{0}$ para $\left.\Sigma_{1}\right)$.

\begin{tabular}{|c|c|c|c|c|c|c|c|c|c|c|c|c|}
\hline & & & & & & & $n=5$ & $L=7$ & & & & \\
\hline & & $a$ & 0 & 0 & 0,5 & 0,5 & 0 & 0,75 & 0,75 & 0 & 1,0 & 1,0 \\
\hline & & $b$ & 0 & 0,5 & 0 & 0,5 & 0,75 & 0 & 0,75 & 1,0 & 0 & 1,0 \\
\hline$c$ & $d$ & - & - & - & - & - & - & - & - & - & - & - \\
\hline \multirow[t]{2}{*}{1,0} & 1,0 & - & 200,0 & $48,9^{*}$ & 49,2 & 19,9 & 16,7 & 16,8 & 5,4 & 6,8 & 6,6 & 2,3 \\
\hline & & - & 200,0 & $36,3 * *$ & 36,3 & 17,4 & 10,8 & 10,8 & 5,1 & 4,3 & 4,3 & 2,3 \\
\hline \multirow[t]{2}{*}{1,25} & 1,0 & - & 42,6 & 22,2 & 17,5 & 11,0 & 11,2 & 8,9 & 4,4 & 5,2 & 4,7 & 2,1 \\
\hline & & - & 28,2 & 15,0 & 12,1 & 8,6 & 7,3 & 6,2 & 3,8 & 3,7 & 3,5 & 2,1 \\
\hline \multirow[t]{2}{*}{1,5} & 1,0 & - & 15,3 & 10,7 & 9,0 & 6,7 & 6,9 & 5,8 & 3,5 & 4,0 & 3,7 & 2,0 \\
\hline & & - & 7,5 & 5,9 & 5,2 & 4,5 & 4,2 & 3,8 & 3,8 & 2,8 & 2,7 & 1,9 \\
\hline \multirow[t]{2}{*}{1,25} & 1,25 & - & 15,6 & 9,9 & 9,8 & 9,8 & 6,2 & 6,3 & 3,5 & 3,9 & 3,9 & 2,0 \\
\hline & & - & 10,2 & 6,7 & 6,7 & 5,0 & 4,4 & 4,4 & 2,9 & 2,9 & 2,9 & 1,9 \\
\hline \multirow[t]{2}{*}{1,5} & 1,5 & - & 4,4 & 3,6 & 3,6 & 3,1 & 3,0 & 3,0 & 2,3 & 2,4 & 2,4 & 1,7 \\
\hline & & - & 3,0 & 2,6 & 2,6 & 2,4 & 2,3 & 2,3 & 1,9 & 1,9 & 1,9 & 1,6 \\
\hline
\end{tabular}

* gráficos conjuntos de $T^{2}$ e $|\mathbf{S}|$; **gráfico MCMAX.

Tabela 3. Valores do NMA - gráficos conjuntos de $T^{2}$ e $|\mathrm{S}|$ e gráfico $\operatorname{MCMAX}\left(\rho=0,5\right.$; matriz de covariâncias muda de $\Sigma_{0}$ para $\left.\Sigma_{1}\right)$.

\begin{tabular}{|c|c|c|c|c|c|c|c|c|c|c|c|c|}
\hline & & & & & & & $n=\mathbf{5}$ & $L=7$ & & & & \\
\hline & & $a$ & 0 & 0 & 0,5 & 0,5 & 0 & 0,75 & 0,75 & 0 & 1,0 & 1,0 \\
\hline & & $b$ & 0 & 0,5 & 0 & 0,5 & 0,75 & 0 & 0,75 & 1,0 & 0 & 1,0 \\
\hline$c$ & $d$ & - & - & - & - & - & - & - & - & - & - & - \\
\hline \multirow[t]{2}{*}{1,0} & 1,0 & - & 200,0 & $38,4^{*}$ & 38,5 & 34,8 & 10,8 & 10,8 & 10,6 & 4,1 & 4,2 & 4,1 \\
\hline & & - & 200,0 & $36,3 * *$ & 36,3 & 17,4 & 10,8 & 10,8 & 5,1 & 4,3 & 4,3 & 2,3 \\
\hline \multirow[t]{2}{*}{1,25} & 1,0 & - & 41,6 & 17,7 & 14,1 & 15,8 & 7,6 & 6,6 & 7,1 & 3,5 & 3,5 & 3,5 \\
\hline & & - & 26,5 & 14,4 & 11,7 & 8,4 & 7,2 & 6,1 & 3,8 & 3,6 & 3,5 & 2,1 \\
\hline \multirow[t]{2}{*}{1,5} & 1,0 & - & 14,6 & 8,9 & 7,7 & 8,4 & 5,2 & 4,6 & 5,0 & 3,0 & 2,9 & 3,0 \\
\hline & & - & 7,1 & 5,7 & 5,0 & 4,3 & 4,1 & 3,7 & 2,8 & 2,7 & 2,7 & 1,9 \\
\hline \multirow[t]{2}{*}{1,25} & 1,25 & - & 15,9 & 8,7 & 8,6 & 8,6 & 4,9 & 4,8 & 4,9 & 2,9 & 2,9 & 2,9 \\
\hline & & - & 10,6 & 6,9 & 6,9 & 5,1 & 4,5 & 4,5 & 3,0 & 2,9 & 2,9 & 1,9 \\
\hline \multirow[t]{2}{*}{1,5} & 1,5 & - & 4,3 & 3,5 & 3,5 & 3,4 & 2,7 & 2,7 & 2,7 & 2,1 & 2,1 & 2,1 \\
\hline & & - & 3,2 & 2,8 & 2,8 & 2,5 & 2,4 & 2,4 & 2,0 & 2,0 & 2,0 & 1,6 \\
\hline
\end{tabular}

* gráficos conjuntos de $T^{2}$ e $|\mathbf{S}| ; * *$ gráfico MCMAX.

Tabela 4. Valores do NMA - gráficos conjuntos de $T^{2}$ e $|\mathrm{S}|$ e gráfico $\operatorname{MCMAX}\left(\rho=0,7\right.$; matriz de covariâncias muda de $\Sigma_{0}$ para $\left.\Sigma_{1}\right)$.

\begin{tabular}{|c|c|c|c|c|c|c|c|c|c|c|c|c|}
\hline & & & & & & & $n=\mathbf{5}$ & $L=7$ & & & & \\
\hline & & $a$ & 0 & 0 & 0,5 & 0,5 & 0 & 0,75 & 0,75 & 0 & 1,0 & 1,0 \\
\hline & & $b$ & 0 & 0,5 & 0 & 0,5 & 0,75 & 0 & 0,75 & 1,0 & 0 & 1,0 \\
\hline$c$ & $d$ & - & - & - & - & - & - & - & - & - & - & - \\
\hline \multirow[t]{2}{*}{1,0} & 1,0 & - & 200,0 & $20,6^{*}$ & 20,2 & 39,7 & 5,7 & 5,6 & 12,9 & 2,3 & 2,3 & 5,0 \\
\hline & & - & 200,0 & $36,3 * *$ & 36,3 & 17,4 & 10,8 & 10,8 & 5,1 & 4,3 & 4,3 & 2,3 \\
\hline \multirow[t]{2}{*}{1,25} & 1,0 & - & 39,1 & 11,9 & 9,8 & 17,1 & 4,6 & 4,2 & 8,2 & 2,2 & 2,2 & 4,1 \\
\hline & & - & 25,1 & 14,0 & 11,4 & 8,2 & 7,1 & 6,0 & 3,8 & 3,6 & 3,6 & 2,1 \\
\hline \multirow[t]{2}{*}{1,5} & 1,0 & - & 13,1 & 6,9 & 5,8 & 8,3 & 3,6 & 3,4 & 5,3 & 2,0 & 2,1 & 3,3 \\
\hline & & - & 6,7 & 5,5 & 4,9 & 4,2 & 4,0 & 3,6 & 2,7 & 2,7 & 2,6 & 1,9 \\
\hline \multirow[t]{2}{*}{1,25} & 1,25 & - & 15,7 & 6,9 & 6,8 & 9,2 & 3,5 & 3,5 & 5,4 & 2,0 & 2,0 & 3,3 \\
\hline & & - & 11,2 & 7,2 & 7,2 & 5,3 & 4,6 & 4,6 & 3,0 & 3,0 & 3,0 & 1,9 \\
\hline \multirow[t]{2}{*}{1,5} & 1,5 & - & 4,3 & 3,1 & 3,1 & 3,6 & 2,3 & 2,3 & 2,9 & 1,7 & 1,7 & 2,2 \\
\hline & & - & 3,4 & 2,9 & 2,9 & 2,6 & 2,5 & 2,5 & 2,0 & 2,0 & 2,0 & 1,6 \\
\hline
\end{tabular}


Tabela 5. Valores do NMA - gráficos conjuntos de $T^{2}$ e $|S|$ e gráfico $\operatorname{MCMAX}\left(\rho=0,5\right.$; matriz de covariâncias muda de $\Sigma_{0}$ para $\left.\Sigma_{1}\right)$.

\begin{tabular}{|c|c|c|c|c|c|c|c|c|c|c|c|c|}
\hline & & & & & & & $n=5$ & $L=7$ & & & & \\
\hline & & $a$ & 0 & 0 & 0,5 & 0,5 & 0 & 0,75 & 0,75 & 0 & 1,0 & 1,0 \\
\hline & & $b$ & 0 & 0,5 & 0 & 0,5 & 0,75 & 0 & 0,75 & 1,0 & 0 & 1,0 \\
\hline$c$ & $d$ & - & - & - & - & - & - & - & - & - & - & - \\
\hline \multirow[t]{2}{*}{1,0} & 1,0 & - & 200,0 & $34,1 *$ & 34,4 & 33,9 & 10,5 & 10,5 & 10,6 & 4,2 & 4,1 & 4,2 \\
\hline & & - & 200,0 & $36,3 * *$ & 36,3 & 17,4 & 10,8 & 10,8 & 5,0 & 4,3 & 4,3 & 2,3 \\
\hline \multirow[t]{2}{*}{1,25} & 1,0 & - & 30,8 & 13,9 & 11,8 & 14,2 & 6,6 & 5,9 & 6,8 & 3,4 & 3,3 & 3,4 \\
\hline & & - & 23,0 & 13,3 & 10,9 & 7,9 & 6,9 & 5,9 & 3,7 & 3,6 & 3,4 & 2,1 \\
\hline \multirow[t]{2}{*}{1,5} & 1,0 & - & 10,2 & 6,9 & 6,3 & 6,9 & 4,4 & 4,0 & 4,4 & 2,7 & 2,8 & 2,8 \\
\hline & & - & 6,5 & 5,3 & 4,8 & 4,2 & 3,9 & 3,5 & 2,7 & 2,7 & 2,6 & 1,9 \\
\hline \multirow[t]{2}{*}{1,25} & 1,25 & - & 10,1 & 6,5 & 6,3 & 6,8 & 4,1 & 4,1 & 4,2 & 2,7 & 2,7 & 2,7 \\
\hline & & - & 8,7 & 6,0 & 6,1 & 4,7 & 4,2 & 4,2 & 2,8 & 2,8 & 2,8 & 1,9 \\
\hline \multirow[t]{2}{*}{1,5} & 1,5 & - & 3,1 & 2,7 & 2,7 & 2,7 & 2,3 & 2,3 & 2,3 & 1,9 & 1,9 & 1,8 \\
\hline & & - & 2,7 & 2,5 & 2,5 & 2,2 & 2,2 & 2,2 & 1,8 & 1,9 & 1,9 & 1,5 \\
\hline
\end{tabular}

* gráficos conjuntos de $T^{2}$ e $|\mathbf{S}|$; ** gráfico MCMAX.

Tabela 6. Valores de X, Y, Z, W e MCMAX (L = 7).

\begin{tabular}{|c|c|c|c|c|c|c|c|c|c|}
\hline Amostra & & & & & & & $Z$ & $W^{*}$ & MCMAX \\
\hline \multirow[t]{2}{*}{1} & $X$ & 1002,47 & 995,36 & 1006,16 & 994,41 & 1001,59 & 0,001 & 0,840 & 0,840 \\
\hline & $Y$ & 1,94 & 1,98 & 2,03 & 2,00 & 2,10 & 0,161 & 0,397 & - \\
\hline \multirow[t]{2}{*}{2} & $X$ & 1000,54 & 998,70 & 1007,74 & 998,71 & 1001,36 & 0,789 & 0,628 & 1,655 \\
\hline & $Y$ & 2,08 & 2,01 & 2,10 & 1,98 & 2,19 & 1,655 & 0,545 & - \\
\hline \multirow[t]{2}{*}{3} & $X$ & 1001,30 & 1001,45 & 997,62 & 998,78 & 999,82 & 0,114 & 0,276 & 0,953 \\
\hline & $Y$ & 2,04 & 2,03 & 2,09 & 1,99 & 2,06 & 0,953 & 0,255 & - \\
\hline \multirow[t]{2}{*}{4} & $X$ & 1004,07 & 1008,04 & 1003,66 & 1001,91 & 998,79 & 1,842 & 0,568 & 1,842 \\
\hline & $Y$ & 1,91 & 2,17 & 1,93 & 1,95 & 1,91 & 0,622 & 0,765 & - \\
\hline \multirow[t]{2}{*}{5} & $X$ & 991,36 & 993,86 & 1000,20 & 1004,11 & 1002,50 & 0,893 & 0,934 & 0,934 \\
\hline & $Y$ & 1,89 & 1,93 & 1,98 & 1,99 & 2,03 & 0,872 & 0,382 & - \\
\hline \multirow[t]{2}{*}{6} & $X$ & 1008,73 & 1001,12 & 1009,29 & 1004,22 & 1002,34 & 2,873 & 0,624 & 2,873 \\
\hline & $Y$ & 2,13 & 1,90 & 2,04 & 2,05 & 1,92 & 0,165 & 0,657 & - \\
\hline \multirow[t]{2}{*}{7} & $X$ & 999,33 & 1006,25 & 994,34 & 1001,33 & 1002,89 & 0,464 & 0,745 & 0,768 \\
\hline & $Y$ & 2,12 & 2,07 & 1,96 & 1,84 & 2,07 & 0,277 & 0,768 & - \\
\hline \multirow[t]{2}{*}{8} & $X$ & 999,31 & 1002,08 & 998,87 & 1011,14 & 1003,55 & 1,672 & 0,834 & 1,672 \\
\hline & $Y$ & 1,84 & 1,94 & 2,02 & 2,09 & 1,92 & 0,832 & 0,647 & - \\
\hline \multirow[t]{2}{*}{9} & $X$ & 1008,73 & 1012,95 & 1009,29 & 1002,22 & 999,34 & 3,637 & 0,938 & 3,637 \\
\hline & $Y$ & 2,07 & 2,03 & 2,05 & 1,94 & 2,02 & 0,487 & 0,325 & - \\
\hline \multirow[t]{2}{*}{10} & $X$ & 1006,93 & 1002,37 & 1004,66 & 1004,32 & 1000,88 & 2,143 & 0,389 & 2,143 \\
\hline & $Y$ & 1,96 & 2,06 & 1,88 & 2,05 & 2,02 & 0,143 & 0,506 & - \\
\hline
\end{tabular}

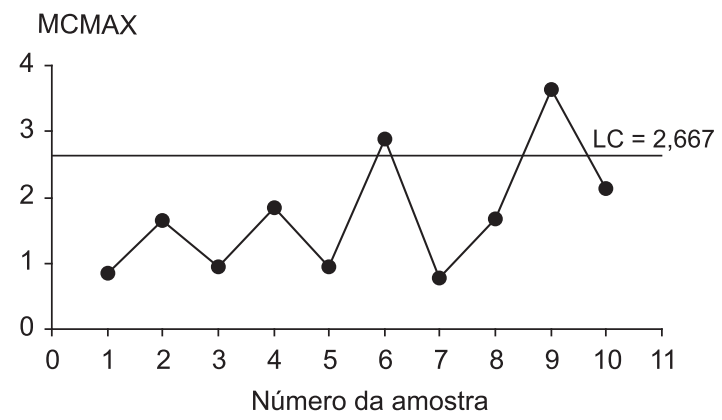

Figura 1. Gráfico de controle MCMAX. para $0,5 / 1,25$. O sinal é dado pela nona amostra que corresponde ao segundo ponto acima do limite de controle, conforme ilustrado na Figura 1. Analisando os dados, confirma-se que a causa especial afetou a variável $X$.

\section{Conclusões}

Neste artigo foi proposto o uso de um único gráfico de controle, isto é, o gráfico MCMAX, para o monitoramento do vetor de médias e da matriz de covariâncias de processos normais bivariados. 
Como o gráfico proposto requer apenas o cálculo de estatísticas já familiares do usuário, isto é, médias e desvios padrões das amostras, ele é muito mais simples de ser utilizado se comparado com os gráficos de $T^{2}$ e $|\mathrm{S}|$. Além disso, com o gráfico proposto, é mais fácil de se saber qual variável foi provavelmente afetada pela causa especial. A adoção da regra de decisão de dois pontos na região de ação concede ao gráfico MCMAX um desempenho geral superior ao dos gráficos conjuntos de $T^{2}$ e $|\mathrm{S}|$, exceto quando a correlação entre as duas características de qualidade é muito alta. Quando a correlação é muito alta, e a causa especial desloca a média e/ou a variabilidade de apenas uma das variáveis, $X$ ou $Y$, os gráficos de $T^{2}$ e $|\mathrm{S}|$ são, em geral, mais ágeis do que gráfico MCMAX.

\section{Agradecimentos}

Os autores agradecem aos dois revisores anônimos os úteis comentários e sugestões. Esta pesquisa contou com o apoio da FAPESP e do CNPq.

\section{Referências}

ALT, F. B. Multivariate quality control. In: KOTZ, S.; JOHNSON, N. L. (Eds.). Encyclopedia of statistical science. New York, NY: John Wiley Sons, 1985. p. 110-122.

CALZADA, M. E.; SCARIANO, S. M. The robustness of the synthetic control chart to non-normality. Communications in Statistics: Simulation and Computation, v. 30, n. 2, p. 311-326, 2001.

CHEN, G.; CHENG, S. W.; XIE, H. A new multivariate control chart for monitoring both location and dispersion. Communications in Statistics-Simulation and Computation, v. 34, n. 1, p. 203-217, 2005.

COSTA, A. F. B.; MACHADO, M. A. G. Synthetic control chart with two-stage sampling for monitoring bivariate processes. Pesquisa Operacional, v. 27, n. 1, p. 117-130, 2007.

COSTA, A. F. B.; MACHADO, M. A. G. A new multivariate control chart for monitoring the covariance matrix of bivariate processes. Communications in StatisticsSimulation and Computation, v. 37, n. 7, p. 1453-1465, 2008.

COSTA, A. F. B.; RAHIM, M. A. A synthetic control chart for monitoring the process mean and variance. Quality in Maintenance Engineering, v. 12, n. 1, p. 1355-2511, 2006.
COSTA, A. F. B.; EPPRECHT E. K.; CARPINETTI, L. C. R. Controle Estatístico de Qualidade. 2 ed. São Paulo: Editora Atlas, 2005. 334 p.

COSTA, A. F. B.; DE MAGALHÃES, M. S.; EPPRECHT, E. K. Monitoring the process mean and variance using synthetic control chart with two-stage testing. International Journal of Production Research, v. 47, n. 18, p. 5067-5086, 2009.

DAVIS, R. B.; WOODALL, W. H. Evaluating and improving the synthetic control chart. Journal of Quality Technology, v. 34, p. 200-208, 2002.

HOTELLING, H. Multivariate quality control, illustrated by the air testing of sample bombsights. In: Techniques of Statistical Analysis. New York: Mc-Graw Hill, 1947. p. 111-184.

KHOO, M. B. C. A new bivariate control chart to monitor the multivariate process mean and variance simultaneously. Quality Engineering, v. 17, p. 109-118, 2005.

MACHADO, M. A. G.; COSTA, A. F. B. The double sampling and the EWMA charts based on the sample variances. International Journal of Production Economics, v. 114, n. 1, p. 134-148, 2008.

MACHADO, M. A. G.; DE MAGALHÃES, M. S.; COSTA, A. F. B. Gráficos de controle de VMAX para o monitoramento da matriz de covariâncias. Produção, v. 18, n. 2, p. 222-239, 2008.

MICROSOFT FORTRAN POWER STATION 4.0. Professional Edition with Microsoft IMSL Mathematical and Statistical Libraries. [S.1.]: Microsoft Corporation, 1995.

MOOD, A. M.; GRAYBILL, F. A.; BOES, D. C. Introduction to the theory of Statistics. McGraw-Hill, 1974.

WU, Z.; SPEDDING, T. A. A synthetic control chart for detecting small shifts in the process mean. Journal of Quality Technology, v. 32, p. 32-38, 2000a.

WU, Z.; SPEDDING, T. A. Implementing synthetic control charts. Journal of Quality Technology, v. 32, p. 75-78, 2000b.

WU, Z.; YEO, S. H.; SPEDDING, T. A. A synthetic control chart for detecting fraction nonconforming increases. Journal of Quality Technology, v. 33, p. 104-111, 2001a.

WU, Z.; ZHANG, X.; YEO, S. H. Design of sum-ofconforming-run-length control charts. European Journal of Operational research, v. 132, p. 187-196, 2001 b.

ZHANG, G.; CHANG, S. I. Multivariate EWMA control charts using individual observations for process mean and variance monitoring and diagnosis. International Journal of Production Research, v. 46, n. 24, p. 6855-6881, 2008. 


\section{Apêndice: Obtenção da probabilidade ps de $W_{x}$ e/ou $W_{y}$ exceder o limite de controle}

Considere as estatísticas

$$
W_{x}=\frac{k S_{x}^{2}}{\sigma_{x}^{2}} \text { e } W_{y}=\frac{k S_{y}^{2}}{\sigma_{y}^{2}} \text {, em que } S_{x}^{2}=\frac{\sum_{i=1}^{n}\left(x_{i}-\bar{X}\right)^{2}}{n-1} \text { e } S_{y}^{2}=\frac{\sum_{i=1}^{n}\left(y_{i}-\bar{Y}\right)^{2}}{n-1} \text { são as variâncias amostrais }
$$

de duas características de qualidade, $X$ e $Y, P_{S}=1-\operatorname{Pr}\left[\left(W_{x}<L C\right) \cap\left(W_{y}<L C\right)\right]$.

Com o processo em controle, a matriz de covariâncias é dada por $\Sigma_{0}=\left(\begin{array}{cc}\sigma_{x}^{2} & \sigma_{x y} \\ \sigma_{y x} & \sigma_{y}^{2}\end{array}\right)$. A causa especial desloca o vetor de médias de $\mu_{0}$ para $\mathrm{m}_{1}^{\prime}=\left(\mu_{\mathrm{x}}+c \sigma_{\mathrm{x}} ; \mu_{\mathrm{y}}+\mathrm{d} \sigma_{\mathrm{y}}\right)$ e/ou altera a matriz de covariâncias de $\Sigma_{0}$ para $\Sigma_{1}=\left(\begin{array}{cc}a^{2} \sigma_{x}^{2} & a b \sigma_{x y} \\ a b \sigma_{x y} & b^{2} \sigma_{y}^{2}\end{array}\right)$ ou para $\Sigma_{2}=\left(\begin{array}{cc}a^{2} \sigma_{x}^{2} & \sigma_{x y} \\ \sigma_{x y} & b^{2} \sigma_{y}^{2}\end{array}\right)$. No primeiro caso, em que $\Sigma_{0}$ se altera para $\Sigma_{1}$, a causa especial não afeta a correlação entre $X$ e $Y$, que é dada por $\rho=\frac{\sigma_{x y}}{\sigma_{x} \sigma_{y}}$.

No segundo caso, no entanto, a causa especial reduz a correlação de $\rho$ para $\frac{\rho}{a b}$. Se X e Y são normalmente distribuídos, e $\left[y_{i}-\left(\mu_{y}+d \sigma_{y}\right)\right] \mid x_{1}, x_{2}, \ldots, x_{n} \sim N\left(\rho \frac{b \sigma_{y}}{a \sigma_{x}}\left[x_{i}-\left(\mu_{x}+c \sigma_{x}\right)\right] ; b^{2} \sigma_{y}^{2}\left(1-\rho^{2}\right)\right)$ ou ainda $\frac{\left[y_{i}-\left(\mu_{y}+d \sigma_{y}\right)\right]+\rho \frac{b \sigma_{y}}{a \sigma_{x}}\left[\left(\mu_{x}+c \sigma_{x}\right)-\overline{X]}\right.}{b \sigma_{y} \sqrt{1-\rho^{2}}} \mid x_{1}, x_{2}, \ldots, x_{n} \sim N\left(\frac{\rho}{\sqrt{1-\rho^{2}}}\left(\frac{x_{i}-\bar{X}}{a \sigma_{x}}\right), 1\right)$.

Como $\rho \frac{b \sigma_{y}}{a \sigma_{x}}\left[\left(\mu_{x}+c \sigma_{x}\right)-\overline{X]}=\left(\mu_{x}+d \sigma_{y}\right)-\bar{Y}\right.$, segue que (ver página 168, MOOD et al., 1974):

$$
\left(\frac{y_{i}-\bar{Y}}{b \sigma_{y}}\right) \frac{1}{\sqrt{1-\rho^{2}}} \mid x_{1}, x_{2}, \ldots, x_{n} \sim N\left(\frac{\rho}{\sqrt{1-\rho^{2}}}\left(\frac{x_{i}-\bar{X}}{a \sigma_{x}}\right), 1\right) \text {. }
$$

Consequentemente,

$\frac{(n-1) S_{y}^{2}}{b^{2} \sigma_{y}^{2} \sqrt{1-\rho^{2}}}\left|x_{1}, x_{2}, \ldots, x_{n}=\frac{(n-1) W_{y}}{k b^{2} \sqrt{1-\rho^{2}}}\right| x_{1}, x_{2}, \ldots, x_{n}=\sum_{i=1}^{n}\left(\frac{y_{i}-\bar{Y}}{b \sigma_{y} \sqrt{1-\rho^{2}}}\right)^{2} \mid x_{1}, x_{2}, \ldots, x_{n} \sim \chi_{n,\left(\rho^{2} / 1-\rho^{2}\right)}^{2} \chi_{n-1}^{2}$ Como $\frac{(n-1) S_{x}^{2}}{a^{2} \sigma_{x}^{2}}=\frac{(n-1) W_{x}}{k a^{2}}=\sum_{i=1}^{n}\left(\frac{x_{i}-\bar{X}}{a \sigma_{x}}\right)^{2} \sim \chi_{n-1}^{2}$ tem-se que

$$
P_{S}=1-\int_{0}^{\frac{(n-1) L C}{k a^{2}}} \operatorname{Pr}\left[\chi_{n,\left(t \rho^{2} / 1-\rho^{2}\right)}^{2}<\frac{(n-1) L C}{k b^{2}\left(1-\rho^{2}\right)}\right] \frac{1}{2^{(n-1) / 2} \Gamma[(n-1) / 2]} e^{-t / 2} t^{[(n-1) / 2]-1} d t
$$

lembrando que a notação $\chi_{n}^{2},\left(\rho^{2} / 1-\rho^{2}\right) \chi_{n}^{2}$ representa uma distribuição de Qui-quadrado com $\mathrm{n}$ graus de liberdade e com o parâmetro de não centralidade dado por $\left(\rho^{2} / 1-\rho^{2}\right) \chi_{n}^{2} \mathrm{tv}$.

A constante de ponderação $\mathrm{k}$ é obtida pela expressão (A1), fazendo $\mathrm{a}=\mathrm{b}=1, \rho=\rho_{0}$ e $P_{s}=1-\sqrt{1-\alpha}$, lembrando que o limite de controle, LC, é obtido da expressão (1). Utilizou-se a sub-rotina CSNDF da biblioteca IMSL FORTRAN (1995) para o cálculo da função de distribuição de qui-quadrado não central. 